\title{
Controlling chaotic transport in two-dimensional periodic potentials
}

\author{
R. Chacón ${ }^{1}$ and A. M. Lacasta ${ }^{2}$ \\ ${ }^{1}$ Departamento de Física Aplicada, Escuela de Ingenierías Industriales, Universidad de Extremadura, Apartado Postal 382, E-06071 \\ Badajoz, Spain \\ ${ }^{2}$ Departament de Física Aplicada, Universitat Politècnica de Catalunya, Avinguda Doctor Marañón 44, E-08028 Barcelona, Spain
}

(Received 25 March 2010; revised manuscript received 13 September 2010; published 7 October 2010)

\begin{abstract}
We uncover and characterize different chaotic transport scenarios in perfect two-dimensional periodic potentials by controlling the chaotic dynamics of particles subjected to periodic external forces in the absence of a ratchet effect (i.e., with no directed transport by symmetry breaking of zero-mean forces). After identifying relevant symmetries of the equations of motion, analytical estimates in parameter space for the occurrence of different transport scenarios are provided and confirmed by numerical simulations. These scenarios are highly sensitive to variations of the system's asymmetry parameters, including the eccentricity of the two-dimensional periodic potential and the direction of dc and ac forces, which could be useful for particle sorting purposes in those cases where chaos is unavoidable.
\end{abstract}

DOI: 10.1103/PhysRevE.82.046207

PACS number(s): 05.45.-a, 05.60.Cd

\section{INTRODUCTION}

Controlling the transport of particles on periodic potential energy surfaces is an old and ubiquitous problem appearing in different fields such as physics, chemistry, and biology [1]. Specific examples include colloidal transport in arrays of optical tweezers [2], flux creep through type-II superconductors [3], and Bose-Einstein condensates with periodic pinning sites [4], among many others. Previous theoretical analysis of the motion of particles on surfaces [5-10] considered mesoscopic models owing to the great complexity of the different transport scenarios. While nonchaotic regimes have been widely studied in the context of noisy overdamped models [11] and the chaotic regime has been mainly considered when directed transport is induced by symmetry breaking (the so-called ratchet effect) $[9,12]$, to the best of our knowledge, the fundamental case of deterministic dissipative chaotic transport (CT) in the absence of a ratchet effect (i.e., with no symmetry breaking of zero-mean forces) has yet received little attention [13]. The study of such a chaotic transport in simple two-dimensional periodic potentials could indeed shed some light on diverse chaotic phenomena of great complexity appearing, for example, in magnetotransport on antidot lattices [14].

The rest of the paper is organized as follows. In the next section we introduce our simple two-dimensional model system and obtain analytical estimates of the regions of the parameter space where chaotic transport can occur by using Melnikov analysis (MA). The analysis of the relevant symmetries of the equations of motion and the chaotic threshold in parameter space is provided in Sec. III, while numerical results confirming the theoretical scenario of chaotic transport are presented in Sec. IV. Finally, Sec. V summarizes our results.

\section{MODEL SYSTEM}

We consider here the classical dynamics of a dissipative particle moving on a standard separable periodic potential, with an external force having both dc and ac components, and neglecting thermal effects:

$$
\begin{aligned}
& m \ddot{x}+\partial V / \partial x=-\mu \dot{x}+f_{0} \cos \theta+f_{1 x} \cos \left(\omega_{x} t\right), \\
& m \ddot{y}+\partial V / \partial y=-\mu \dot{y}+f_{0} \sin \theta+f_{1 y} \cos \left(\omega_{y} t\right),
\end{aligned}
$$

where an overdot denotes a derivative with respect to $t, \theta$ describes the direction of the dc force $\mathbf{f}_{0}, \mu$ is the phenomenological coefficient of friction, and $V(x, y)$ $=V_{0}\left[\cos \left(2 \pi x / \lambda_{x}\right)+\cos \left(2 \pi y / \lambda_{y}\right)\right] / 2$ is the potential with $\lambda_{x}, \lambda_{y}$ being the characteristic length scales. A main purpose of the present work is a theoretical characterization of the different CT scenarios by providing analytical estimates of the threshold conditions in parameter space by using MA.

\section{A. Melnikov analysis}

We now briefly describe this method for the simple case of a perturbed integrable Hamiltonian system with one degree of freedom. Consider the system

$$
\begin{gathered}
\mathbf{x}_{t}=h_{0}(\mathbf{x})+\varepsilon h_{1}(\mathbf{x}, t), \\
\mathbf{x}=\left(x_{1}, x_{2}\right),
\end{gathered}
$$

where the unperturbed system $(\varepsilon=0)$ is an integrable Hamiltonian system which possesses a hyperbolic fixed point $X_{0}$ and a separatrix orbit $x_{0}(t)$ such that $\lim _{t \rightarrow \pm \infty} x_{0}(t)=X_{0}$, while the stable and unstable manifolds $x^{s}(t), x^{u}(t)$ are smoothly joined. Generally, the perturbation term $h_{1}$ can introduce dissipation and nonautonomous excitation, with $h_{1}$ being $T$ periodic in time. For $\varepsilon \neq 0$, the perturbed stable and unstable manifolds no longer join smoothly such that, if the ratio of dissipation and excitation is sufficiently small, the stable and unstable manifolds will intersect transversally, creating a homoclinic point. This process is called a homoclinic bifurcation and indicates the onset of chaotic instabilities. To check when a transverse crossing occurs, Melnikov introduced a function [now being known as the Melnikov function (MF)] which measures the distance between the perturbed stable and unstable manifolds in the Poincare section. If the MF presents a simple zero, the manifolds intersect transversally and chaotic instabilities result. See Refs. [15-17] for more details about MA. 


\section{B. Melnikov function}

For the sake of a dimensionless description, we put the equations of motion (1) and (2) into the form

$$
\begin{gathered}
\ddot{r}_{x}+\sin r_{x}=-\gamma \dot{r}_{x}+F_{0 x} \cos \theta+F_{1 x} \cos \left(\Omega_{x} \tau\right), \\
\ddot{r}_{y}+\frac{\sin r_{y}}{a^{2}}=-\gamma \dot{r}_{y}+\frac{F_{0 x}}{a} \sin \theta+\frac{F_{1 x} b}{a} \cos \left(c \Omega_{x} \tau\right),
\end{gathered}
$$

where all variables and parameters are dimensionless, an overdot denotes a derivative with respect to $\tau$ $\equiv \pi t\left(2 V_{0} / m\right)^{1 / 2} / \lambda_{x}, \quad r_{x} \equiv 2 \pi x / \lambda_{x} \pm \pi, \quad r_{y} \equiv 2 \pi y / \lambda_{y} \pm \pi, \quad \gamma$ $\equiv \mu \lambda_{x}\left(2 m V_{0}\right)^{-1 / 2} / \pi, \quad F_{0 x} \equiv f_{0} \lambda_{x} /\left(\pi V_{0}\right), \quad F_{1 x} \equiv \lambda_{x} f_{1 x} /\left(\pi V_{0}\right)$, $\Omega_{x} \equiv \omega_{x} \lambda_{x}\left[m /\left(2 V_{0}\right)\right]^{1 / 2} / \pi, \quad a \equiv \lambda_{y} / \lambda_{x}, \quad b \equiv f_{1 y} / f_{1 x}, \quad$ and $c$ $\equiv \omega_{y} / \omega_{x}$. It is also assumed that the system [Eqs. (4) and (5)] satisfies the MA requirements, i.e., the dissipation and forcing terms are small-amplitude perturbations of the underlying conservative pendulum $\ddot{r}_{x, y}+\sin r_{x, y}=0$. Straightforward application of MA to Eqs. (4) and (5) yields the MFs

$$
\begin{gathered}
M_{x}^{ \pm}\left(\tau_{0}\right)=D_{x}^{ \pm} \pm 2 \pi F_{1 x} \operatorname{sech}\left(\frac{\pi \Omega_{x}}{2}\right) \cos \left(\Omega_{x} \tau_{0}\right), \\
M_{y}^{ \pm}\left(\tau_{0}\right)=D_{y}^{ \pm} \pm 2 \pi a b F_{1 x} \operatorname{sech}\left(\frac{\pi a c \Omega_{x}}{2}\right) \cos \left(c \Omega_{x} \tau_{0}\right),
\end{gathered}
$$

respectively, where the positive (negative) sign refers to the top (bottom) homoclinic orbit of the conservative pendulum, and $D_{x}^{ \pm} \equiv \pm 2 \pi F_{0 x} \cos \theta-8 \gamma, D_{y}^{ \pm} \equiv \pm 2 \pi a F_{0 x} \sin \theta-8 a \gamma$. Since the MFs (6) and (7) have an infinity of simple zeros, a main conclusion is that necessary conditions for the onset of chaotic instabilities are, respectively,

$$
\begin{gathered}
F_{1 x}>\frac{\min \left\{\left|D_{x}^{+}\right|,\left|D_{x}^{-}\right|\right\}}{2 \pi} \cosh \left(\frac{\pi \Omega_{x}}{2}\right), \\
F_{1 x}>\frac{\min \left\{\left|D_{y}^{+}\right|,\left|D_{y}^{-}\right|\right\}}{2 \pi a b} \cosh \left(\frac{\pi a c \Omega_{x}}{2}\right) .
\end{gathered}
$$

Next, one can compare the theoretical predictions and Lyapunov exponent (LE) calculations [16] with the caveat that one cannot expect a too good quantitative agreement between the two kinds of results because LE provides information concerning solely steady chaos, while MA is a perturbative method generally related to transient chaos [17]. To quantify the sorting capability associated with the threshold of chaotic transport, we evaluate the Cartesian components of the velocity, $\left\langle v_{i}\right\rangle=\lim _{\tau \rightarrow \infty}\left\langle r_{i}(\tau)\right\rangle / \tau(i=x, y)$, where angular brackets indicate average over initial conditions, and construct the velocity components parallel and perpendicular to the external dc force $\mathbf{f}_{0},\left\langle v_{\|}\right\rangle=\left\langle v_{x}\right\rangle \cos \theta+a\left\langle v_{y}\right\rangle \sin \theta$ and $\left\langle v_{\perp}\right\rangle=-\left\langle v_{x}\right\rangle \sin \theta+a\left\langle v_{y}\right\rangle \cos \theta$, respectively. We characterize the deviation of $\langle\mathbf{v}\rangle$ from $\mathbf{f}_{0}$ by means of the quantifier

$$
\tan \alpha=\left\langle v_{\perp}\right\rangle /\left\langle v_{\|}\right\rangle,
$$

where $\alpha$ is the deflection angle [10]. For the sake of clarity, we shall consider here the case with equal frequencies $(c=1)$ and both dc and ac forces acting on the same direction $\left(f_{1 x} \equiv f_{1} \cos \theta, f_{1 y} \equiv f_{1} \sin \theta\right.$, and hence $\left.b=\tan \theta\right)$. By defining $F_{1} \equiv \lambda_{x} f_{1} /\left(\pi V_{0}\right)$, one has $F_{1 x}=F_{1} \cos \theta$, and hence Eqs. (8) and (9) reduce to

$$
\begin{gathered}
F_{1}>F_{1, t h}^{x} \equiv \frac{\min \left\{\left|D_{x}^{+}\right|,\left|D_{x}^{-}\right|\right\}}{2 \pi|\cos \theta|} \cosh \left(\frac{\pi \Omega_{x}}{2}\right), \\
F_{1}>F_{1, t h}^{y} \equiv \frac{\min \left\{\left|D_{y}^{+}\right|,\left|D_{y}^{-}\right|\right\}}{2 \pi a|\sin \theta|} \cosh \left(\frac{\pi a \Omega_{x}}{2}\right),
\end{gathered}
$$

respectively, where $F_{1, t h}^{x}, F_{1, t h}^{y}$ are the chaotic threshold amplitudes.

\section{SYMMETRY ANALYSIS}

Equations (11) and (12) tell us that the onset of chaos in both directions strongly depends on the external force direction $\theta$, which can thus be used as a high-sensitivity control parameter to suppress and strengthen CT in one or another direction at will. Specifically, one straightforwardly obtains from Eqs. (11) and (12) that the chaotic threshold amplitudes exhibit (as functions of $\theta$ ) the symmetries

$$
\begin{gathered}
F_{1, t h}^{x}(\pi / 2 \pm \theta)=F_{1, t h}^{x}(\pi / 2 \mp \theta), \\
F_{1, t h}^{y}(\pi / 2 \pm \theta)=F_{1, t h}^{y}(\pi / 2 \mp \theta), \\
F_{1, t h}^{x}(\pi / 4 \pm \theta)=F_{1, t h}^{y}(\pi / 4 \mp \theta), \\
F_{1, t h}^{x}(3 \pi / 4 \pm \theta)=F_{1, t h}^{y}(3 \pi / 4 \mp \theta) .
\end{gathered}
$$

Now, the following remarks are in order. First, symmetries (13)-(16) follow directly from the symmetries of the equations of motion [Eqs. (4) and (5)] with respect to the angles $\theta=\pi / 2, \pi / 4$. Second, Eqs. (13) and (14) are valid for any spatial potential $(a>0)$, while Eqs. (15) and (16) are solely valid for a symmetric potential $(a=1)$. Third, symmetries (15) and (16) imply that different transport regimes are expected in the $x$ and $y$ directions, as the external force direction deviates from the "symmetric" angles $\pi / 4$ and $3 \pi / 4$, respectively. Fourth, symmetries (15) and (16) also imply $\left\langle v_{x}\right\rangle(\pi / 4 \pm \theta)=\left\langle v_{y}\right\rangle(\pi / 4 \mp \theta)$ and $\left\langle v_{x}\right\rangle(3 \pi / 4 \pm \theta)=\left\langle v_{y}\right\rangle(3 \pi / 4 \mp \theta)$, respectively, and hence $\tan \alpha$ (as a function of $\theta$ ) exhibits the symmetry

$$
\begin{gathered}
\tan \alpha(\pi / 4+\theta)=-\tan \alpha(\pi / 4-\theta), \\
\tan \alpha(3 \pi / 4+\theta)=-\tan \alpha(3 \pi / 4-\theta),
\end{gathered}
$$

i.e., for a symmetric potential, $\tan \alpha$ is an odd function of $\theta$ with respect to the angles $\pi / 4$ and $3 \pi / 4$, respectively. Note that this is no longer the case for an asymmetric potential according to the second remark. It is worth mentioning that, in the absence of ac forces $\left(F_{1 x}=0\right)$ and $a=1$, the deflection of (nonchaotic) particles still exhibits the aforementioned symmetries with respect to the angles $\pi / 4$ and $3 \pi / 4$ since the first remark holds for such a limiting case. In particular, when $F_{0 x}>1, F_{1 x}=0$, and $\gamma$ is sufficiently high the only 
attractors of Eqs. (4) and (5) are limit cycles corresponding to rotations [18].

\section{NUMERICAL RESULTS}

Extensive numerical simulations confirmed all the above theoretical predictions. Thus, by varying $\theta$ one can find different transport regimes (see Fig. 1, top panel): CT in both directions (as for $\theta=\{2 \pi / 9,5 \pi / 18\}$ ), CT in one direction while intermittent periodic transport (PT) in the other (as for $\theta=\{\pi / 6, \pi / 3\})$, PT in both directions (as for $\theta$ $=\{7 \pi / 36, \pi / 4,11 \pi / 36\})$, and PT in one direction while periodic oscillation in the other (as for $\theta=\{13 \pi / 36,5 \pi / 36\}$ ). Since the onset of chaos also depends on the particle mass [through the coefficient of friction; cf. Eqs. (11) and (12)], such a $\theta$ dependence can therefore be used to sort different particles according to their mass. For two kinds of particles with different masses, this means that one can obtain analytical estimates of the optimal force directions $\theta_{o p t}$ from Eqs. (11) and (12) such that one particle exhibits CT while the other does not, with the remaining parameters being held constant. Numerical experiments confirmed this scenario as is shown in Fig. 1 (middle panel) and Fig. 2. Additionally, the onset of chaos also depends on the eccentricity parameter $a$ [Eq. (12)]: decreasing or increasing $a$ from 1 (symmetric potential) means increasing the potential's asymmetry. Thus, the eccentricity of the periodic potential can also be used as an effective parameter to control CT on a periodic surface, as in the case of optical potentials, for example [19]. Figure 1 (bottom panel) shows an illustrative example where typical trajectories are plotted for increasing values of $a$ from 1 . Starting at a situation where CT occurs in both directions $(a=1)$, one finds that increasing the potential's asymmetry $(a>1)$ changes the motion to PT in both directions (as for $a=1.2$ ). This behavior changes again to CT in both directions for higher values of $a$ (as for $a=1.4$ ), and finally changes to $\mathrm{PT}$ in the $x$ direction while remain bounded inside a well in the $y$ direction (as for $a=1.6$ ). Also, the effectiveness of a fixed external force at sorting heavy particles is enhanced by breaking the potential symmetry (recall that $\gamma \sim m^{-1 / 2}$; see Fig. 2). Figure 3 shows illustrative instances of maximal LEs, $\Lambda_{x}^{+}$and $\Lambda_{y}^{+}$, which quantify the chaotic dynamics in the $x$ and $y$ directions, respectively, versus $\theta$ for two values of the eccentricity parameter. According to the first remark, these diagrams present exactly the same symmetries than those of the chaotic threshold amplitudes [Eqs. (13)-(16), respectively]:

$$
\begin{gathered}
\Lambda_{x}^{+}(\pi / 2 \pm \theta)=\Lambda_{x}^{+}(\pi / 2 \mp \theta), \\
\Lambda_{y}^{+}(\pi / 2 \pm \theta)=\Lambda_{y}^{+}(\pi / 2 \mp \theta), \\
\Lambda_{x}^{+}(\pi / 4 \pm \theta)=\Lambda_{y}^{+}(\pi / 4 \mp \theta), \\
\Lambda_{x}^{+}(3 \pi / 4 \pm \theta)=\Lambda_{y}^{+}(3 \pi / 4 \mp \theta),
\end{gathered}
$$

i.e., Eqs. (19) and (20) are valid for any spatial potential (a $>0$ ), while Eqs. (21) and (22) are solely valid for a symmetric potential $(a=1)$. One typically finds how different chaotic
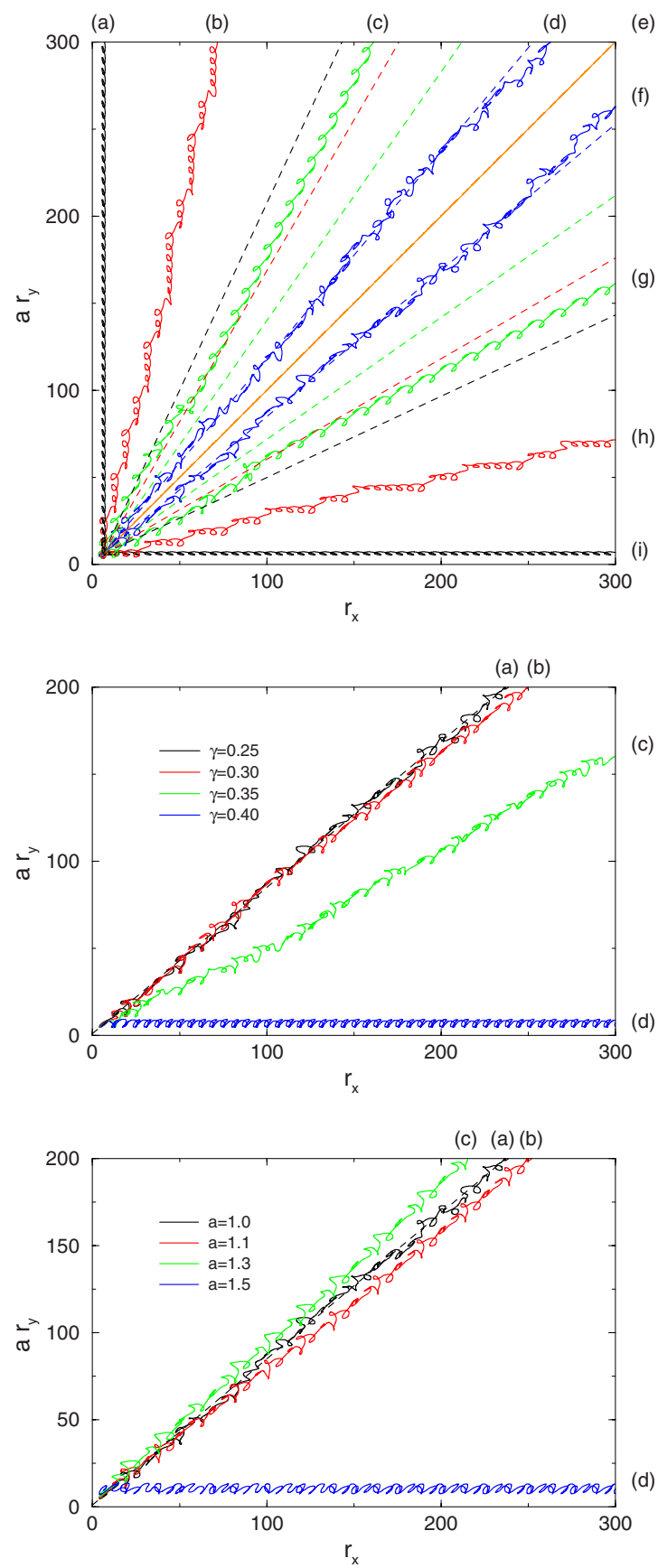

FIG. 1. (Color online) Top panel: trajectories for a net force applied at different angles: $\theta=13 \pi / 36$ (a), $\pi / 3$ (b), $11 \pi / 36$ (c), $5 \pi / 18$ (d), $\pi / 4$ (e), $2 \pi / 9$ (f), $7 \pi / 36$ (g), $\pi / 6$ (h), and $5 \pi / 36$ (i) for $a=1$ and $\gamma=0.25$. Middle panel: trajectories for $a=1, \theta=2 \pi / 9$, and four values of the dimensionless coefficient of friction: $\gamma$ $=0.25$ (a), 0.3 (b), 0.35 (c), and 0.4 (d). Bottom panel: trajectories for $\theta=2 \pi / 9, \gamma=0.25$, and four values of the eccentricity parameter: $a=1$ (a), 1.1 (b), 1.3 (c), and 1.5 (d). Other parameters are $F_{0 x}=0.28, F_{1}=1$, and $\Omega_{x}=0.68$. Dotted lines indicate the direction of the external force.

and nonchaotic regimes drastically change over certain $\theta$ ranges as the potential becomes asymmetric. For instance, PT in both directions at $\theta=\{\pi / 4,3 \pi / 4\}$ for a symmetric potential $(a=1)$ changes to $\mathrm{CT}$ in solely one direction for an asymmetric potential ( $a=1.5)$ (cf. Fig. 3). Finally, numerical 


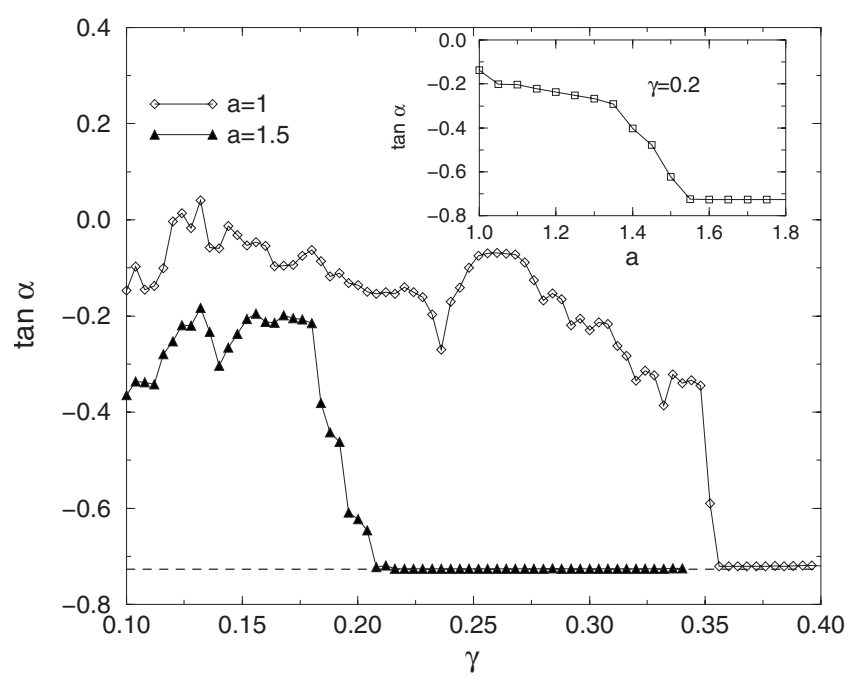

FIG. 2. Deflection angle vs coefficient of friction for two values of the eccentricity parameter: $a=1(\diamond)$ and $1.5(\mathbf{\Delta})$. The inset shows the deflection angle vs eccentricity parameter for $\gamma=0.2$. Other parameters are $\theta=\pi / 5, F_{0 x}=0.28, F_{1}=1, \Omega_{x}=0.68$. The solid lines are solely plotted to guide the eye.

simulations confirmed the accuracy of predictions (17) and (18) as is shown in Fig. 4. Starting with CT in both directions at $\theta=\pi / 4$ for a symmetric potential (Fig. 4, top panel), one sees that the deflection of particles increases as $\theta$ deviates from $\pi / 4$ according to the route described in Fig. 1, top panel. The maximum deflection occurs at symmetric angles with respect to $\pi / 4, \theta_{\max }^{\text {low }}, \theta_{\max }^{\mathrm{sup}}\left(\pi / 4-\theta_{\max }^{\text {low }} \simeq \theta_{\max }^{\mathrm{sup}}-\pi / 4\right)$, where there is PT in one direction while periodic oscillation in the other. For $\theta \geq \theta_{\max }^{\text {sup }}\left[\theta \leq \theta_{\max }^{\text {low }}\right]$, this transport regime remains, i.e., $\left\langle v_{x}\right\rangle=0\left[\left\langle v_{y}\right\rangle=0\right]$, and hence $\tan \alpha\left(\theta \geq \theta_{\max }^{\text {sup }}\right)$ $=\cot \theta\left[\tan \alpha\left(\theta \leq \theta_{\text {max }}^{\text {low }}\right)=-\tan \theta\right.$ ] [cf. Eq. (10)]. For an asymmetric potential (Fig. 4, bottom panel), the dependence of the deflection angle on the external force direction essentially presents a similar scenario to that of the symmetric case, but now $\tan \alpha$ is no longer an odd function with respect $\pi / 4$, as predicted (cf. fourth remark). Finally, it is worth noting that

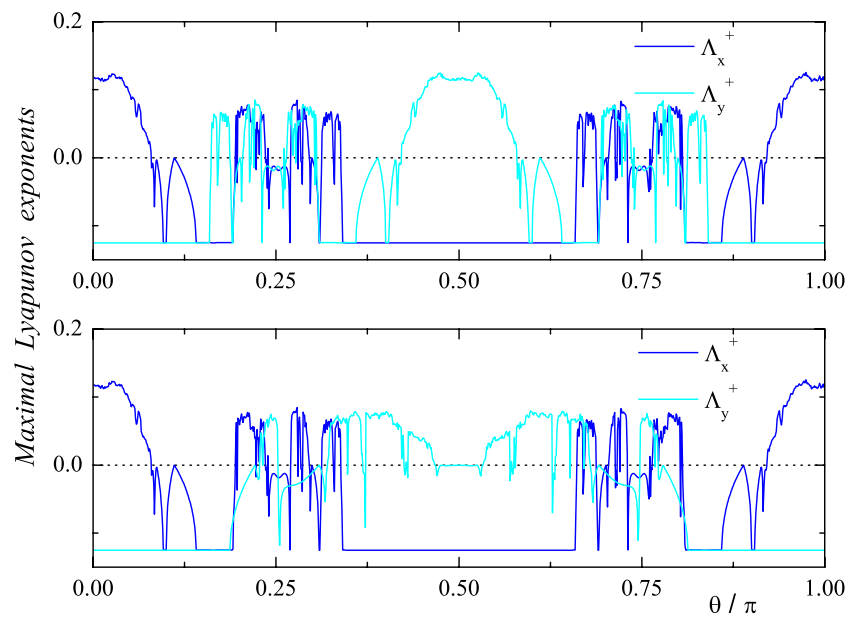

FIG. 3. (Color online) Maximal LEs $\Lambda_{x}^{+}$(dark gray) and $\Lambda_{y}^{+}$ (light gray) as functions of the angle $\theta$ for two values of the eccentricity parameter: $a=1$ (top panel) and 1.5 (bottom panel). Other parameters are $F_{0 x}=0.28, F_{1}=1, \gamma=0.25$, and $\Omega_{x}=0.68$.
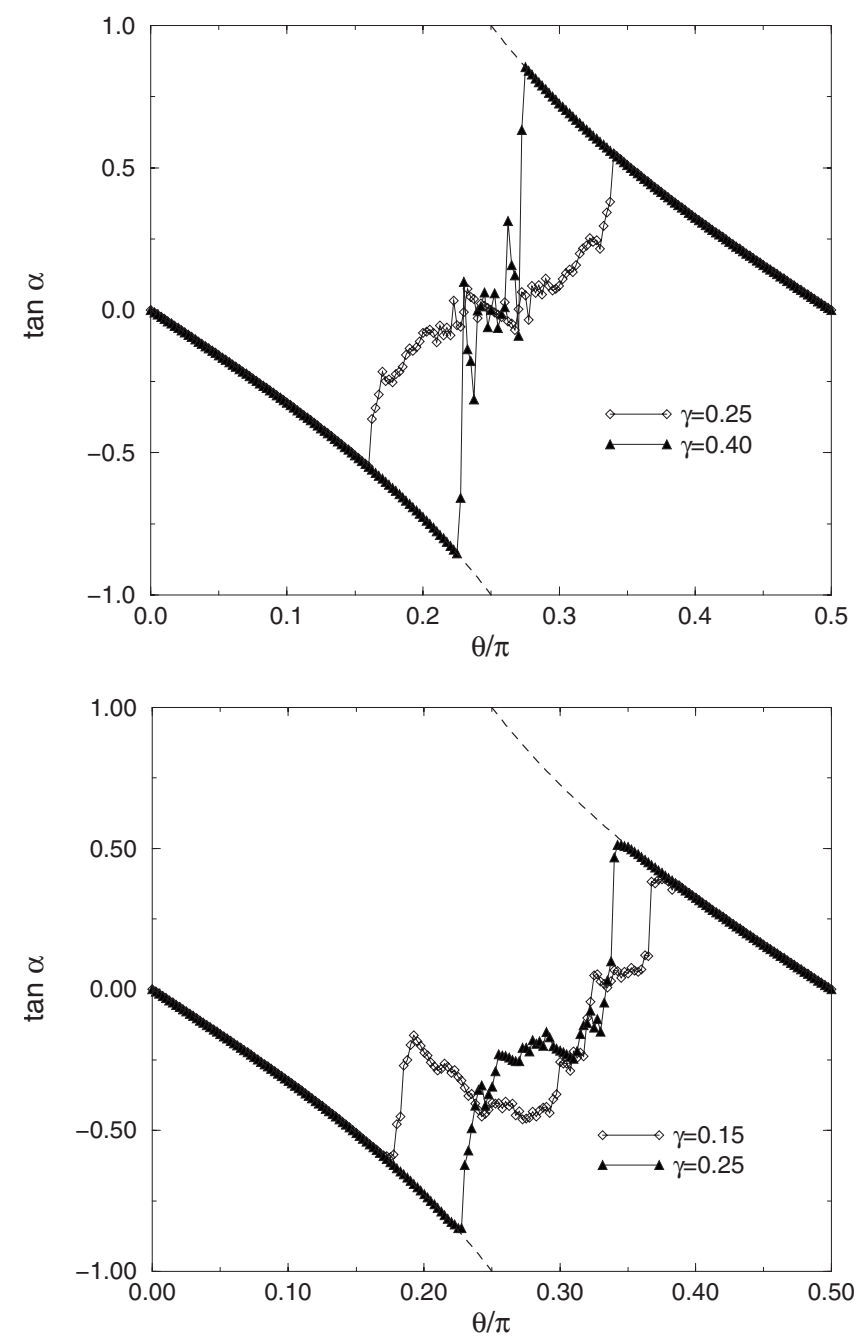

FIG. 4. Deflection angle vs external force direction for $F_{0 x}$ $=0.28, F_{1}=1, \Omega_{x}=0.68$; two values of the eccentricity parameter: $a=1$ (top panel) and 1.5 (bottom panel); and different values of the coefficient of friction: $\gamma=0.25(\diamond), 0.4(\boldsymbol{\Delta})$ (top panel) and $\gamma$ $=0.15(\diamond), 0.25(\mathbf{\Delta})$ (bottom panel). Also plotted are the functions $-\tan \theta$ and $\cot \theta$ (dashed lines; see the text).

the above transport scenarios are intrinsically associated with a chaotic dynamics in the sense that for $F_{1 x}=0$ there is neither chaos [cf. Eqs. (8) and (9)] nor transport for any value of the angle $\theta$ and the remaining fixed parameters.

\section{CONCLUSIONS}

To sum up, we have demonstrated theoretically and numerically through a simple and general system that reliable control of sorting in two-dimensional periodic potentials is achieved for chaotic particles by identifying the relevant symmetries of the equations of motion and the chaotic threshold in parameter space. We uncovered and characterized different sorting scenarios associated with symmetric and asymmetric spatial potentials, which could motivate experiments in different contexts such as optical and antidot lattices. Among the most interesting extensions of this work are the case with the ac and dc forces having different directions, where preliminary results indicate the presence of in- 
triguing "absolute negative mobility" phenomena [20], as well as the study of the effect of noise on the present transport scenarios: even very small amounts of noise may cause both a transition from a bounded state to a running state and a significant modification of the chaotic threshold in parameter space [21].

\section{ACKNOWLEDGMENTS}

We thank Katja Lindenberg for useful discussions. This work was partially supported by the Ministerio de Ciencia e Innovación (MCINN, Spain) under Projects No. FIS200801383 (R.C.) and No. FIS2009-13360-C03-03 (A.M.L.).
[1] H. Risken, The Fokker-Planck Equation (Springer, Berlin, 1984), Chap. 11.

[2] P. T. Korda, M. B. Taylor, and D. G. Grier, Phys. Rev. Lett. 89, 128301 (2002).

[3] C. Reichhardt and F. Nori, Phys. Rev. Lett. 82, 414 (1999).

[4] J. W. Reijnders and R. A. Duine, Phys. Rev. Lett. 93, 060401 (2004).

[5] A. W. Ghosh and S. V. Khare, Phys. Rev. Lett. 84, 5243 (2000).

[6] J. D. Bao and Y. Z. Zhuo, Phys. Lett. A 239, 228 (1998).

[7] I. Derényi and R. D. Astumian, Phys. Rev. E 58, 7781 (1998).

[8] R. Eichhorn, P. Reimann, and P. Hänggi, Phys. Rev. Lett. 88, 190601 (2002).

[9] R. Guantes and S. Miret-Artés, Phys. Rev. E 67, 046212 (2003).

[10] A. M. Lacasta, J. M. Sancho, A. H. Romero, and K. Lindenberg, Phys. Rev. Lett. 94, 160601 (2005).

[11] J. P. Gleeson, J. M. Sancho, A. M. Lacasta, and K. Lindenberg, Phys. Rev. E 73, 041102 (2006).

[12] S. Denisov, Y. Zolotaryuk, S. Flach, and O. Yevtushenko,
Phys. Rev. Lett. 100, 224102 (2008).

[13] P. Hänggi and F. Marchesoni, Rev. Mod. Phys. 81, 387 (2009).

[14] M. Khoury, A. M. Lacasta, J. M. Sancho, A. H. Romero, and K. Lindenberg, Phys. Rev. B 78, 155433 (2008), and references therein.

[15] V. K. Melnikov, Trans. Mosc. Math. Soc. 12, 1 (1963).

[16] See, e.g., A. J. Lichtenberg and M. A. Lieberman, Regular and Chaotic Dynamics (Springer, New York, 1992), Chaps. 5 and 7.

[17] J. Guckenheimer and P. J. Holmes, Nonlinear Oscillations, Dynamical Systems, and Bifurcations of Vector Fields (Springer, Berlin, 1983).

[18] M. Levi, F. Hoppensteadt, and W. Miranker, Q. Appl. Math. 35, 167 (1978).

[19] G. Grynberg and C. Robilliard, Phys. Rep. 355, 335 (2001).

[20] D. Speer, R. Eichhorn, and P. Reimann, Phys. Rev. Lett. 102, 124101 (2009).

[21] P. J. Martínez and R. Chacón, Phys. Rev. Lett. 93, 237006 (2004). 